\title{
Correlações Genéticas e Fenotípicas entre Características de Conformação e Produção de Leite em Bovinos da Raça Pardo-Suíça no Brasil
}

\author{
Francisco Palma Rennó ${ }^{1}$, Cláudio Vieira de Araújo ${ }^{2}$, José Carlos Pereira ${ }^{3}$, \\ Marcelo Silva de Freitas ${ }^{1}$, Robledo de Almeida Torres ${ }^{3}$, Luciana Navajas Rennó ${ }^{4}$, José \\ Augusto Gomes Azevêdo ${ }^{5}$, Fernando da Rocha Kaiser 6
}

\begin{abstract}
RESUMO - Foram avaliados dados de classificação para tipo fornecidos pela Associação Brasileira de Criadores de Gado PardoSuíço com o objetivo de avaliar os efeitos de fatores não-genéticos, estimar a herdabilidade e as correlações genéticas e fenotípicas das características lineares de tipo, características de classificação e a classificação final, bem como avaliar as correlações das características de tipo com a produção de leite. Para o estudo dos fatores não-genéticos foi utilizado o método dos quadrados mínimos, utilizando-se o procedimento GLM do SAS, e, para a estimativa da herdabilidade e correlações genéticas e fenotípicas, foi utilizado um modelo animal. Os componentes de variância e (co)variância foram obtidos com o programa MTDFREML (Multiple Trait Derivative Free Restricted Maximum Likelihood), utilizando a metodologia de máxima verossimilhança restrita livre de derivadas. Os efeitos não-genéticos influenciaram a avaliação da maioria das características de tipo, devendo ser considerados nas avaliações de conformação de bovinos desta raça. As estimativas de herdabilidade obtidas sugerem a possibilidade de ganhos genéticos moderados advindos da seleção para características de tipo. Devido às altas correlações genéticas entre as características lineares de tipo, programas de melhoramento para características de conformação podem ser implementados sem a necessidade de inclusão de todas as características. As correlações genéticas observadas entre algumas características de tipo e a produção de leite sugerem a participação destas na formação de índices de seleção para animais desta raça.
\end{abstract}

Palavras-chave: características de tipo, parâmetros genéticos, Pardo-Suíço

\section{Genetic and Phenotypic Correlations Among Type Traits and Milk Yield of Brown Swiss Cattle in Brazil}

\begin{abstract}
Data of type classification from Brown Swiss Cattle Breeder's Association of Brazil were used to evaluate non-genetic factors, heritability and genetic and phenotypic correlations of the linear type traits, classification traits and final score, as well as to evaluate correlations of type traits and milk yield. For the study of non-genetic factors was utilized the least square metolology using the GLM procedure of SAS, and, for estimate of heritability and genetic and phenotypic correlation was used an animal model. The REML metodology was used to obtain variance and covariance components to estimate heritability and genetic and phenotypic correlations. The non-genetic factors influenced most of the type traits and should be considered in the type evaluation of cattle of this breed. Heritability estimates suggest the possibility of moderate genetic gain for selection of type traits. Genetic correlations among some type traits were high, suggesting that some of them could be excluded from the used classification system. The genetic correlations between some type traits and milk yield suggest the participation of these in the elaboration of selection index of animals of this breed.
\end{abstract}

Key Words: Brown Swiss, genetic parameters, type traits

\section{Introdução}

Ênfase primária em programas de seleção de rebanhos leiteiros é o aumento da produção de leite, devido a, usualmente, vacas de maiores produções de leite serem mais lucrativas (Bertrand et al., 1985). No entanto, a seleção para aumento da produção de leite, por si só, pode levar à diminuição do mérito de características de conformação e afetar a saúde de vacas leiteiras, diminuindo a longevidade e elevando a taxa de descarte involuntário, com conseqüente redução da lucratividade com estes animais (Rogers \& McDaniel, 1989; Short \& Lawlor, 1992; Van Dorp et al, 1998).

\footnotetext{
1 Doutorando em Zootecnia, DZO/UFV. E.mail: fprenno@alunos.ufv.br

2 Professor da Faculdade de Ciências Agrárias do Pará - FCAP. E.mail: araujocv@bol.com.br

3 Professor do Departamento de Zootecnia/UFV, Bolsista do CNPq. E.mail: jcarlos@ufv.br, rtorres@ufv.br

4 Professora do Departamento de Zootecnia da Universidade Estadual do Oeste do Paraná - UNIOESTE

5 Professor do DCAA/ Universidade Estadual de Santa Cruz.

6 Superintendente Técnico da Associação Brasileira dos Criadores de Gado Pardo-Suíço.
} 
Quando são obtidos aumentos na longevidade de vacas leiteiras, são esperadas melhorias gerais na saúde, produção e reprodução, o que resulta em aumento da lucratividade de rebanhos leiteiros, devido ao menor número de novilhas de reposição necessárias à substituição das vacas descartadas, a oportunidade dos custos de criação de novilhas serem dispersos por um período de tempo mais longo, e também pelo aumento do número de vacas produzindo na idade adulta (Rogers et al., 1988; Allaire \& Gibson, 1992).

A longevidade ou vida produtiva de vacas leiteiras pode ser definida como o intervalo de tempo entre o primeiro parto e o descarte da vaca do rebanho (Van Raden \& Klaaskate, 1993). Van Vleck \& Norman (1972) citam que os maiores argumentos que favorecem a seleção para componentes de tipo são que determinadas características lineares estariam positivamente correlacionadas com a longevidade, ou, paralelamente, poderiam indicar os motivos de descarte de vacas leiteiras.

Para a avaliação das características de tipo foi introduzido, a partir de 1983, o sistema de classificação linear, que é baseado em uma escala biológica contínua, constituída de informações sobre a classificação final, características de classificação e mais de 13 características lineares de tipo (Thompson et al., 1983). Este sistema de classificação é adotado pela maioria das associações de criadores de raças de bovinos leiteiros para a avaliação das características de conformação (Norman et al., 1988).

Utilizando o sistema de classificação linear, Weigel et al (1998) avaliaram as relações entre as características lineares de tipo e a longevidade, verificando que as características de sistema mamário e a angulosidade apresentaram as maiores correlações genéticas com a longevidade $(0,22$ a 0,32 , e 0,41 , respectivamente), resultados semelhantes aos verificados por Rogers et al. (1989), Boldman et al. (1992) e Short \& Lawlor (1992).

Dessa forma, a utilização das características de tipo e da produção de leite na implementação de critérios de seleção em rebanhos leiteiros poderia resultar em aumentos da produção leiteira e, ao mesmo tempo, resultar em menor taxa de descarte involuntário e maior número de dias de vida produtiva das vacas leiteiras (Weigel et al, 1998).

Diversos estudos têm evidenciado a importância do conhecimento das correlações genéticas e fenotípicas entre as características lineares de tipo, e também, entre as características lineares de tipo e as características produtivas, principalmente a produção de leite. Quando são verificadas as conclusões resultantes da maioria dos trabalhos que avaliaram as correlações entre características lineares de tipo e a produção de leite, observa-se que estas correlações são de moderada magnitude e, portanto, com aplicação prática em programas de seleção (Short \& Lawlor, 1992; Mrode \& Swanson, 1994; Esteves, 1999).

Para estimativa das correlações genéticas e fenotípicas entre as características de tipo e a produção de leite, estima-se a herdabilidade destas características. Nos estudos em que foi estimada a herdabilidade da produção de leite, foram encontrados valores de 0,31 a 0,37 , segundo os trabalhos de Short \& Lawlor (1992), Campos et al. (1994) e Rennó et al. (2001). Para as características lineares de tipo, Misztal et al. (1992) encontraram coeficientes de herdabilidade que variaram de 0,10 a 0,42 , sendo a estatura a característica que apresentou maior valor de herdabilidade.

Klassen et al. (1992) verificaram valores semelhantes, obtendo coeficientes de herdabilidade das características de tipo variando de 0,07 a 0,48. A maioria das características tem herdabilidade moderada, entre 0,10 a 0,25 , com as características estatura, abertura e ângulo da garupa apresentando os maiores valores e, ângulo do casco, inserção do úbere anterior e textura do úbere, apresentando os menores valores. Resultados semelhantes foram obtidos por Boettcher et al. (1998) e Gengler et al. (1999).

As estimativas de correlações genéticas e fenotípicas entre as características lineares de tipo são importantes no sentido de considerar ou não a necessidade da inclusão de algumas delas nas avaliações dos sistemas de classificação linear. Usualmente, as correlações fenotípicas têm sido inferiores as correlações genéticas entre as diversas características de tipo (Klassen et al., 1992; Brotherstone et al., 1994).

Segundo Mrode \& Swanson (1994), as maiores correlações genéticas e fenotípicas entre as características lineares de tipo envolveram as características relacionadas à capacidade corporal e, também, entre as características do sistema mamário. As maiores correlações negativas foram verificadas entre as características de pernas e pés. Klassen et al. (1992) e Short \& Lawlor (1992) também verificaram correlações genéticas e fenotípicas positivas, na sua maioria, entre as características lineares de tipo. 
As correlações fenotípicas entre as características de tipo e a produção de leite são geralmente baixas, próximas de zero (Short \& Lawlor, 1992; Norman et al., 1996). As maiores correlações fenotípicas encontradas foram observadas entre a produção de leite e a largura do úbere posterior, a altura do úbere posterior, a característica leiteira e a pontuação final (Norman et al., 1996; McManus \& Saueressig, 1998; Esteves, 1999).

Com relação às correlações genéticas entre as características de tipo e a produção de leite, Norman et al. (1988), Short \& Lawlor (1992), Misztal et al. (1992) e Esteves (1999), verificaram que características lineares de tipo como característica leiteira, largura do úbere posterior, altura do úbere posterior, e pontuação final, geralmente apresenta correlações genéticas positivas, de moderada a alta, com a produção de leite. No entanto, características como profundidade de úbere e inserção de úbere anterior, segundo os autores citados anteriormente, são negativamente correlacionadas com a produção de leite.

Grande parte dos estudos de avaliação das características de conformação em vacas leiteiras se refere a trabalhos realizados em outros países, como os Estados Unidos, Canadá e Holanda, e invariavelmente com animais da raça Holandesa. No Brasil, poucos trabalhos foram realizados, senão os estudos de McManus \& Saueressig (1998) e Esteves (1999), ambos avaliando animais da raça Holandesa.

Desta forma, o objetivo deste estudo foi obter estimativas de parâmetros genéticos e verificar as correlações genéticas e fenotípicas entre as características de tipo e a produção de leite em bovinos da raça Pardo-Suíça no Brasil.

\section{Material e Métodos}

Os dados utilizados neste estudo são provenientes do Programa de Classificação Linear da Associação Brasileira dos Criadores de Gado Pardo-Suíço (ABCGPS), sendo fornecidos através do convênio mantido entre o Departamento de Zootecnia da Universidade Federal de Viçosa e a ABCGPS. Os dados de produção de leite foram provenientes dos arquivos do Serviço de Controle Leiteiro da ABCGPS.

O arquivo de classificação utilizado é constituído de 1379 observações, incluindo 15 características lineares descritivas, cinco características de classificação e a pontuação final (Tabela 1), com datas de classificação nos anos de 1993 a 2001, referentes à avaliação de 14 classificadores, de acordo com o sistema de classificação linear adotado pela ABCGPS (ABCGPS, 2000). Foram avaliados animais de 97 rebanhos, compreendendo progênies de 111 reprodutores, com o arquivo de pedigree contendo 5415 animais. Quanto à produção de leite, 436 informações foram utilizadas, e se referem a lactações encerradas no mesmo período.

As lactações estudadas foram ajustadas previamente a partir de fatores de ajustamento multiplicativos, segundo Rennó (2001). Os fatores de ajustamento referem-se ao número de ordenhas diárias, sendo corrigidas para duas ordenhas (2x), duração da lactação, para 305 dias (305-d), e para produção a idade adulta da vaca (IAV). Foram consideradas duas épocas de classificação, época 1, que corresponde aos meses de abril a setembro de cada ano; e época 2, que corresponde aos meses de outubro a março do ano subseqüente.

A idade da vaca a classificação variou de 22 a 152 meses, sendo incluída no modelo como efeito fixo. Foram formadas classes da idade da vaca a classificação: 1) vacas avaliadas com idade inferior a 30 meses; 2) vacas avaliadas de 30 a 36 meses; 3) vacas avaliadas de 36 meses a 48 meses; e 4) vacas avaliadas com idade superior a 48 meses.

Foi incluído o estágio de lactação na avaliação das características de tipo, variando de 3 a 400 dias de lactação, também sendo incluído como efeito fixo no modelo utilizado. Foram formadas oito classes de estágios de lactação a classificação: 1) animais de 3 a 30 dias de lactação; 2) animais de 31 a 60 dias de lactação; 3) animais de 61 a 90 dias de lactação; 4) animais de 91 a 150 dias de lactação; 5) animais de 151 a 180 dias de lactação; 6) animais de 181 a 220 dias de lactação; 7) animais de 221 a 280 dias de lactação; e 8) animais de 281 a 400 dias de lactação. Foi também exigido que cada reprodutor possuísse no mínimo duas filhas, com número de filhas por reprodutor variando de 2 a 184, e que cada rebanho pertencente a este arquivo tivesse ao menos três animais classificados por ano.

Foi utilizado o método dos quadrados mínimos, utilizando o procedimento GLM do SAS (SAS,1991), avaliando os efeitos fixos de rebanho, ano e época de classificação, classificador, idade da vaca e estágio de lactação a classificação, e o efeito aleatório de reprodutor sobre as características lineares, as características de classificação e a classificação final. O modelo matemático adotado é descrito abaixo: 
$\hat{Y}_{i j k l m n o p}=\mu+\mathrm{r}_{\mathrm{i}}+a e_{(j k)}+c_{l}+i_{m}+l_{n}+t_{o}+\varepsilon_{i j k l m n o p}$

em que: $\mathrm{Y}_{\mathrm{ijklmnop}}$ é a variável resposta da vaca p; no i-ésimo rebanho; no j-ésimo ano de classificação, na k-ésima época de classificação, do l-ésimo classificador, da m-ésima idade da vaca a classificação, do n-ésimo estágio de lactação a classificação, do o-ésimo reprodutor; $\mu$, a média geral da característica estudada; $r_{i}$, o efeito fixo do rebanho $i$; $a_{j}$, efeito fixo de ano de classificação j; $e_{k}$, o efeito fixo da época de classificação k; cl, efeito fixo do classificador l; $\mathrm{i}_{\mathrm{m}}$, o efeito fixo da idade da vaca a classificação; $l_{n}$, efeito fixo do estagio de lactação a classificação; $\mathrm{t}_{0}$, efeito aleatório de reprodutor pai da vaca; eijklmnop, efeito aleatório residual associado a cada observação, com média 0 e variância $\sigma_{\mathrm{e}}{ }^{2}$.

Os componentes de variância e covariâncias para as estimativas de parâmetros genéticos e correlações genéticas e fenotípicas foram obtidas com o programa MTDFREML (Multiple Trait Derivative Free Restricted Maximum Likelihood), descrito por Boldman et al. (1995), utilizando a metodologia de máxima verossimilhança restrita livre de derivadas. Para a avaliação das características de tipo foi utilizado modelo animal, considerando os efeitos fixos de rebanho, ano-época de classificação, classificador, estágio de lactação e idade do animal a classificação. Para a avaliação da produção de leite corrigida, foram considerados os efeitos fixos de rebanho, ano e época de partos.

Para o cálculo das herdabilidades, as variâncias genética aditiva, residual e fenotípica foram obtidas em análise de características simples. Para o cálculo das correlações genéticas e fenotípicas e residuais as variâncias e covariâncias foram obtidas pela análise das características aos pares (duas a duas).

\section{Resultados e Discussão}

Os valores médios e os respectivos desviospadrão das características lineares de tipo, das características de classificação, da classificação final e da produção de leite ajustada para duas ordenhas, 305 dias de lactação e a idade adulta da vaca, podem ser observados na Tabela 1.

As médias das características de conformação se apresentam semelhantes aos resultados obtidos por Misztal et al. (1992) e Short \& Lawlor (1992), com animais da raça Holandesa, e Norman et al. (1996), com animais da raça Pardo-Suíça. A maioria das características apresentou diferença média, entre estes estudos, de \pm 3 pontos, com exceção da profundidade de úbere e do ligamento central, onde os resultados do presente trabalho foram cerca de 5 pontos superior e 5 pontos inferior, respectivamente, nestas características, quando comparadas com o estudo de Norman et al. (1996).

A média de produção de leite verificada (Tabela 1) é superior a obtida por Rennó (2001), provavelmente devido aos anos estudados no presente trabalho e no estudo citado anteriormente, diferirem. Porém, quando as médias de produção deste estudo são comparadas com as médias obtidas nos anos finais do estudo de Rennó (2001), estas são semelhantes.

O resumo da análise de variância das características de tipo podem ser observadas nas Tabelas 2 e 3 . Os efeitos de rebanho e ano-época de classificação, sendo relativos aos grupos contemporâneos, influenciaram quase todas as características lineares. No entanto, na avaliação das características de classificação e a classificação final, somente o rebanho influenciou estas características, com o efeito de ano-época de classificação influenciando somente a pontuação da característica pernas e pés e sistema mamário. A importância deste efeito é esperada, segundo Esteves (1999), refletindo, principalmente, diferenças de manejo, alimentação, aspectos sanitários e condições climáticas entre os diferentes rebanhos nos anos avaliados.

De forma semelhante, o efeito de classificador foi significativo para quase todas as características lineares, para todas as características de classificação e para a classificação final, sendo resultado semelhante aos obtidos por Klassen et al. (1992).

Este efeito poderia ser explicado em função dos técnicos da ABCGPS geralmente classificarem os mesmos rebanhos, principalmente por razões de localização dos criatórios e da região de atuação dos técnicos ser determinada pela facilidade de acesso. Desta forma, o efeito do classificador sobre as características de conformação reflete, de forma mais contundente, as diferenças existentes entre critérios de seleção entre rebanhos do que de critérios de avaliação entre os classificadores.

O efeito de idade da vaca a classificação foi fonte de variação significante para as características lineares de capacidade corporal (estatura, força, profundidade corporal, angulosidade, largura de 
Tabela 1 - Número de observações (N), média não ajustadas, desvio-padrão (D.P.), pontuações mínimas (Min.) e máximas (Max.) e coeficientes de variação (C.V.) das características lineares de tipo, características de classificação, classificação final e produção de leite ajustada para duas ordenhas, 305 dias de lactação e idade adulta da vaca

Table 1 - Number of observations (N), not adjusted means, standard deviation (D.P.), maximum (Max.) and minimum (Min.) scores, coefficient of variation (C.V.) of linear type traits, classification traits, final score and milk yield adjusted for two milking daily, 305 days of lactation period and mature age

\begin{tabular}{|c|c|c|c|c|c|c|}
\hline $\begin{array}{l}\text { Característica } \\
\text { Trait } \\
\end{array}$ & $\mathrm{N}$ & $\begin{array}{l}\text { Média } \\
\text { Mean }\end{array}$ & D.P. & Min. & Max. & $\mathrm{CV}$ \\
\hline Classificação Final (Final score) & 1357 & 83,47 & 3,98 & 66 & 92 & 4,77 \\
\hline \multicolumn{7}{|l|}{ Características de Classificação (Classification traits) } \\
\hline Formato (Frame) & 1332 & 83,61 & 4,37 & 67 & 90 & 5,23 \\
\hline Característica leiteira (Dairy character) & 1331 & 84,31 & 4,14 & 67 & 90 & 4,92 \\
\hline Capacidade corporal (Body capacity) & 1332 & 84,21 & 4,25 & 67 & 90 & 5,06 \\
\hline Pernas e pés (Feet and legs) & 1208 & 84,15 & 4,15 & 67 & 90 & 4,93 \\
\hline \multirow[t]{2}{*}{ Sistema mamário (Mammary system) } & 1332 & 83,01 & 4,51 & 67 & 90 & 5,44 \\
\hline & \multicolumn{6}{|c|}{$\begin{array}{c}\text { Características lineares } \\
\text { Linear type traits }\end{array}$} \\
\hline Estatura (Stature) & 1379 & 26,45 & 7,32 & 5 & 48 & 27,68 \\
\hline Força (Strength) & 1379 & 25,84 & 5,80 & 5 & 45 & 22,45 \\
\hline Profundidade corporal (Body deep) & 1379 & 29,90 & 6,07 & 5 & 50 & 20,33 \\
\hline Angulosidade (Dairy form) & 1379 & 31,21 & 6,32 & 10 & 48 & 20,25 \\
\hline Ângulo da garupa (Rump angle) & 1379 & 24,56 & 5,49 & 5 & 50 & 22,38 \\
\hline Abertura da garupa (Thurl width) & 1379 & 23,99 & 5,51 & 5 & 45 & 22,97 \\
\hline Pernas traseiras (vista lateral) (Rear legs - side view) & 1379 & 26,83 & 4,64 & 5 & 45 & 17,29 \\
\hline Ângulo do casco (Foot angle) & 1379 & 26,00 & 4,62 & 12 & 45 & 17,77 \\
\hline Inserção do úbere anterior (Fore udder attachment) & 1379 & 26,83 & 8,25 & 5 & 49 & 30,77 \\
\hline Altura do úbere posterior (Rear udder heigth) & 1379 & 28,00 & 7,33 & 5 & 50 & 26,19 \\
\hline Largura do úbere posterior (Rear udder width) & 1379 & 28,42 & 9,35 & 5 & 50 & 32,92 \\
\hline Ligamento central (Udder cleft) & 1379 & 20,49 & 7,05 & 5 & 45 & 34,41 \\
\hline Profundidade do úbere (Udder deep) & 1379 & 34,69 & 8,04 & 5 & 50 & 23,19 \\
\hline Colocação de tetas (Teat placement) & 1379 & 26,17 & 5,74 & 5 & 48 & 21,95 \\
\hline \multirow[t]{2}{*}{ Comprimento de tetas (Teat length) } & 1379 & 26,54 & 5,84 & 10 & 50 & 22,00 \\
\hline & \multicolumn{6}{|c|}{$\begin{array}{c}\text { Produção de leite } \\
\text { Milkyield }\end{array}$} \\
\hline Produção de leite (Milk yield) & 436 & 7214,65 & 1768,97 & 3179 & 13621 & 24,52 \\
\hline
\end{tabular}

garupa), pernas e pés, e de sistema mamário (largura de úbere posterior, ligamento central, profundidade de úbere e comprimento de tetas). Também influenciaram as características de classificação e a classificação final, com exceção da característica pernas e pés.

Segundo Bowden (1982) e Cue et al. (1996), este efeito é esperado sobre as características de tipo devido ao aumento da idade de vacas leiteiras, onde algumas características melhoram, enquanto outras pioram. Na Tabela 4, estão as médias de classificação final e das características de classificação em função da idade da vaca à classificação.

O estágio de lactação influenciou todas as características lineares de sistema mamário, com exceção da inserção de úbere anterior, e, ao mesmo tempo, não influenciou as características lineares de pernas e pés e de capacidade corporal, com exceção da força. As características de classificação, formato e capacidade corporal, foram afetadas pelo estágio de lactação, enquanto que as demais e a classificação final não foram afetadas. Analisando isoladamente algumas características de sistema mamário, observa-se tendência de maiores pontuações para animais que foram classificados no início de lactação, no entanto, estas diferenças não são observadas quando o sistema mamário é analisado como um todo. Nos estudos de McManus \& Saueressig (1998) e Esteves (1999) são observados resultados semelhantes.

O efeito de reprodutor pai da vaca foi significativo para diversas características lineares de tipo, bem como para as características de classificação e para a classificação final. Este efeito indica variação entre 
Tabela 2 - Resumo das análises de variâncias das características lineares de tipo em função dos efeitos fixos de rebanho (REB), ano-época de classificação (AECL), classificador (CLAS), idade da vaca a classificação (IVC), estágio de lactação a classificação (LAC), e efeito aleatório de reprodutor pai da vaca (TOU)

Table 2 - Summary of analysis of variance for type traits with agreement of fixed effects of herd (HER), year-season of classification (AECL), classifier (CLAS), age of cow at classification (IVC), stage of lactation at classification (LAC), and random effect of sire (TOU)

\begin{tabular}{|c|c|c|c|c|c|c|}
\hline & \multicolumn{6}{|c|}{$\begin{array}{c}\text { Quadrados Médios } \\
\text { Means squares }\end{array}$} \\
\hline & REB & AECL & CLAS & IVC & LAC & TOU \\
\hline $\operatorname{EST}(S T A)$ & $77,53^{* *}$ & $141,98 * *$ & $71,38 *$ & $994,15^{* *}$ & 59,03 & $56,84 * *$ \\
\hline FOR (STR) & $45,95 * *$ & $54,04 * *$ & $120,40 * *$ & $599,95 * *$ & $71,14 * *$ & $29,13^{*}$ \\
\hline $\operatorname{PRO}(B D)$ & $54,41^{* *}$ & 24,32 & 42,10 & $1555,78 * *$ & 29,62 & $34,76^{*}$ \\
\hline $\mathrm{ANG}(D F)$ & $44,44^{* *}$ & $91,66^{* *}$ & 48,36 & $988,06 * *$ & 46,05 & $43,88 * *$ \\
\hline $\mathrm{AGA}(R A)$ & $36,60 * *$ & 26,20 & $60,02 * *$ & 50,23 & 16,80 & $38,11^{* *}$ \\
\hline LGA $(T W)$ & $42,15^{* *}$ & $72,66 * *$ & $221,75^{* *}$ & $396,81^{* *}$ & 20,90 & 19,34 \\
\hline $\operatorname{PTR}(R L)$ & 25,81 & $31,00 *$ & 26,78 & $206,23^{* *}$ & 18,98 & 21,14 \\
\hline $\operatorname{ANC}(F A)$ & $28,31 * *$ & $54,54 * *$ & $50,55 * *$ & $50,24^{*}$ & 14,48 & 18,13 \\
\hline IAU (FUA) & $96,83^{* *}$ & $132,26 * *$ & $204,24 * *$ & 120,42 & 18,07 & 48,90 \\
\hline AUP (RUH) & $45,09 * *$ & $345,18 * *$ & $440,27 * *$ & 15,07 & $62,71 * *$ & 29,92* \\
\hline LUP (RUW) & $135,25^{* *}$ & $236,55^{* *}$ & $247,77 * *$ & $1654,48 * *$ & $177,05^{* *}$ & $69,65^{*}$ \\
\hline $\mathrm{LIC}(U C)$ & $52,44 * *$ & $116,11^{* *}$ & $184,02 * *$ & $130,00 * *$ & $217,77 * *$ & 37,69 \\
\hline PRU (UD) & $95,25 * *$ & $209,11^{* *}$ & $166,84 * *$ & $2327,67 * *$ & $122,21 * *$ & $52,91^{*}$ \\
\hline CTE (TP) & $33,31^{*}$ & 27,89 & $138,03 * *$ & 23,41 & $60,32 *$ & $32,50 *$ \\
\hline CMT (TL) & $33,33 * *$ & 14,68 & $85,95 * *$ & $202,32 * *$ & $50,56^{*}$ & $34,28 * *$ \\
\hline
\end{tabular}

EST=estatura; FOR=força; PRO=profundidade corporal; ANG=angulosidade; AGA=ângulo da garupa; LGA=abertura da garupa;

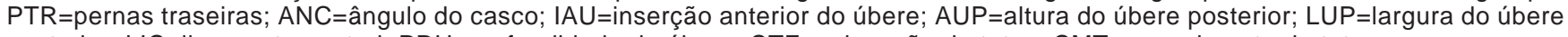
posterior; LIC=ligamento central; PRU=profundidade de úbere; CTE=colocação de tetas; CMT=comprimento de tetas.

${ }^{1}$ Em todas as características lineares, são 93 graus de liberdade para REB, 14 para AECL, 11 para classificador, 3 para IVC, 7 para LAC e 108 para TOU.

$S T A=$ stature; STR=strength; $B D=$ body deep; $D F=$ dairy form; $R A=$ rump angle; $T W=$ thurl width; $R L=$ rear legs; $F A=$ foot angle; FUA=fore udder attachment; $R U H=$ rear udder heigth; $R U W=$ rear udder width; $U C=$ udder cleft; $U D=$ udder deep; $T P=$ teat placement; $T L=$ teat length.

${ }^{1}$ For overall linear type traits is 93 degree of freedom for REB, 14 for AECL, 11 for CLAS, 3 for IVC, 7 for LAC and 108 for TOU.

** $\mathrm{P}<0,01$ e * $\mathrm{P}<0,05$.

as progênies dos touros e que, provavelmente, as características de tipo apresentam algum componente genético aditivo.

As estimativas de herdabilidade obtidas neste estudo foram geralmente de moderada à alta, estando descritas na Tabela 5. O resultado apresentado se refere à análise das características de tipo e produção de leite como característica única, estando também descritas as amplitudes dos coeficientes de herdabilidade obtidos nas análises de duas características simultâneas. Todos os coeficientes de herdabilidade estimados como característica única estão dentro das amplitudes dos coeficientes obtidos na estimativa da herdabilidade na análise de duas características simultâneas.

A estimativa de herdabilidade da produção de leite como característica única foi de 0,22, estando também, dentro das amplitudes estimadas que variaram de 0,18 a 0,25 . Este resultado é semelhante aos verificados por Matos et al. (1997) e Freitas et al. (1999), entretanto é inferior aos valores estimados por Campos et al. (1994) e Rennó et al. (2001).
Os coeficientes de herdabilidade para as características lineares de tipo variaram entre 0,02 e 0,39, com as características estatura $(0,39)$, colocação de tetas $(0,29)$ e força $(0,19)$ apresentando os maiores coeficientes de herdabilidade. As características profundidade corporal $(0,09)$, pernas traseiras (vista lateralmente) $(0,09)$ e ângulo do casco $(0,02)$ apresentaram os menores valores nas estimativas de herdabilidade das características lineares de tipo. Diferentemente do usualmente obtido na literatura, para a característica linear largura de garupa foi estimado coeficiente de herdabilidade igual a zero, indicando que as variações ocorridas poderiam ser atribuídas aos efeitos de meio e efeitos genéticos não aditivos.

Para as características de classificação as estimativas de herdabilidade variaram de 0,07 (pernas e pés) a 0,20 (característica leiteira), refletindo os valores obtidos nas características lineares que compõe a pontuação em cada característica de classificação, segundo o sistema de classificação adotado na ABCGPS. A classificação final apresentou estimati- 
Tabela 3 - Resumo das análises de variâncias das características de classificação e da classificação final, em função dos efeitos fixos de rebanho (REB), ano-época de classificação (AECL), classificador (CLAS), idade da vaca a classificação (IVC), estágio de lactação a classificação (LAC), e efeito aleatório de reprodutor pai da vaca (TOU)

Table 3 - Summary of analysis of variance for classification traits and final score, in agreement of fixed effects of herd (REB), yearseason of classification (AECL), classifier (CLAS), age of cow at classification (IVC), stage of lactation at classification $(L A C)$, and random effect of sire (TOU)

\begin{tabular}{|c|c|c|c|c|c|c|}
\hline & \multicolumn{6}{|c|}{$\begin{array}{c}\text { Quadrados médios } \\
\text { Mean squares }\end{array}$} \\
\hline & REB & AECL & CLAS & IVC & LAC & TOU \\
\hline & \multicolumn{6}{|c|}{$\begin{array}{c}\text { Características de classificação }^{1} \\
\text { Classification traits }\end{array}$} \\
\hline FRM (FR) & $20,44 * *$ & 15,48 & $62,30 * *$ & $361,89 * *$ & $25,45^{*}$ & $15,39 *$ \\
\hline CLE $(D F)$ & $17,38 * *$ & 16,10 & $73,12 * *$ & $235,57 * *$ & 11,11 & $18,70 * *$ \\
\hline $\mathrm{PCO}(B C)$ & $21,34 * *$ & 15,89 & $78,11^{* *}$ & $279,64 * *$ & $24,09 *$ & $14,73^{* *}$ \\
\hline $\mathrm{PPE}^{2}(F L)$ & $15,22 *$ & $23,07 *$ & $68,09 * *$ & 3,84 & 13,12 & 12,49 \\
\hline \multirow[t]{2}{*}{ SMA (MS) } & $24,48 * *$ & $26,15^{* *}$ & $78,24 * *$ & $139,17^{* *}$ & 13,69 & $20,12 * *$ \\
\hline & \multicolumn{6}{|c|}{$\begin{array}{c}\text { Classificação final }^{2} \\
\text { Final score }\end{array}$} \\
\hline CLF (FS) & $17,52^{* *}$ & $13,11 *$ & $76,88 * *$ & $173,34 * *$ & 3,16 & $11,37 * *$ \\
\hline \multicolumn{7}{|c|}{ 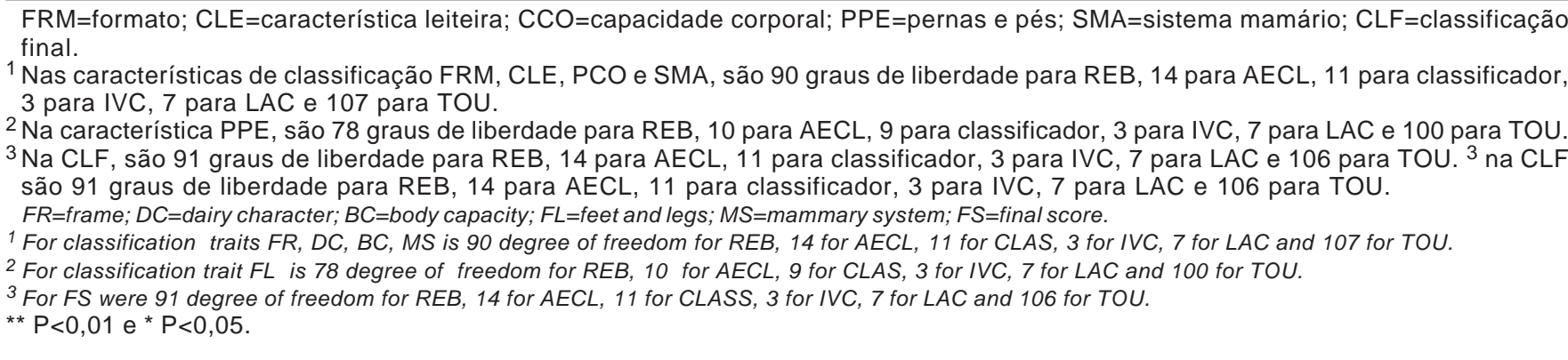 } \\
\hline
\end{tabular}

Tabela 4 - Classificação final (CLF) e características de classificação formato (FRM), característica leiteira (CLE), capacidade corporal (CCO), pernas e pés (PPE) e sistema mamário (SMA), segundo a idade da vaca a classificação (IVC)

Table 4 - Final score (CLF) e classification traits frame (FRM), dairy character (CLE), body capacity (CCO), feet and legs (PPE) and mammary system (SAM), according de age of cow at classification (IVC)

\begin{tabular}{|c|c|c|c|c|c|c|}
\hline \multirow[t]{2}{*}{ IVC } & \multicolumn{6}{|c|}{$\begin{array}{c}\text { Característica } \\
\text { Trait } \\
\end{array}$} \\
\hline & CLF & FRM & CLE & $\mathrm{CCO}$ & PPE & SMA \\
\hline $\begin{array}{l}1 \text { (até } 30 \text { meses) } \\
\text { until } 30 \text { months }\end{array}$ & $83,22^{b}$ & $83,06^{\mathrm{b}}$ & $83,81^{b}$ & $83,33^{b}$ & 84,29 & $82,98^{b}$ \\
\hline $\begin{array}{l}2(30-36 \text { meses }) \\
30-36 \text { months }\end{array}$ & $83,04^{b}$ & $82,96^{\mathrm{b}}$ & $83,76^{b}$ & $83,75^{b}$ & 84,16 & $82,48^{\mathrm{b}}$ \\
\hline $\begin{array}{l}3 \text { ( } 36 \text { a } 48 \text { meses) } \\
36-48 \text { months }\end{array}$ & $82,97^{b}$ & $83,12^{b}$ & $84,06^{b}$ & $83,85^{b}$ & 83,48 & $82,50^{b}$ \\
\hline $\begin{array}{l}4 \text { (+ } 48 \text { meses) } \\
+36 \text { months }\end{array}$ & $84,86^{a}$ & $85,56^{\mathrm{a}}$ & $85,80^{a}$ & $86,08^{\mathrm{a}}$ & 84,91 & $84,36^{\mathrm{a}}$ \\
\hline
\end{tabular}

Médias seguidas de letras iguais não diferem, a 5\% de probabilidade, pelo teste Tukey.

Values within column with different letters significantly differ $(P<.05)$ by Tukey test. 
Tabela 5 - Estimativa de herdabilidade $\left(h^{2}\right)$ das características lineares de tipo, das características de classificação e da classificação final obtidas em análises com característica única e amplitude das estimativas

Table 5 - Heritability $\left(h^{2}\right)$ of linear type traits, classification traits and final score obtained from unique trait analyses and estimates amplitude

\begin{tabular}{|c|c|c|}
\hline $\begin{array}{l}\text { Característica } \\
\text { Traits }\end{array}$ & $\mathrm{h}^{2}$ & $\begin{array}{c}\text { Amplitude }^{1} \\
\text { Amplitude }\end{array}$ \\
\hline Classificação Final (Final score) & 0,18 & $0,14-0,23$ \\
\hline \multicolumn{3}{|l|}{ Características de Classificação (Classification traits) } \\
\hline Formato (Frame) & 0,13 & $0,12-0,23$ \\
\hline Característica leiteira (Dairy character) & 0,20 & $0,18-0,27$ \\
\hline Capacidade corporal (Body capacity) & 0,12 & $0,11-0,15$ \\
\hline Pernas e pés (Feet and legs) & 0,07 & $0,06-0,10$ \\
\hline \multirow[t]{2}{*}{ Sistema mamário (Mammary system) } & 0,17 & $0,13-0,19$ \\
\hline & \multicolumn{2}{|c|}{$\begin{array}{c}\text { Características lineares } \\
\text { Linear type traits }\end{array}$} \\
\hline Estatura (Stature) & 0,39 & $0,36-0,40$ \\
\hline Força (Strength) & 0,19 & $0,18-0,21$ \\
\hline Profundidade corporal (Body deep) & 0,09 & $0,08-0,10$ \\
\hline Angulosidade (Dairy form) & 0,17 & $0,15-0,21$ \\
\hline Ângulo da garupa (Rump angle) & 0,18 & $0,17-0,19$ \\
\hline Abertura de garupa (Thurl width) & 0,00 & $0,00-0,01$ \\
\hline Pernas traseiras (vista lateral) (Rear legs - side view) & 0,09 & $0,05-0,10$ \\
\hline Ângulo do casco (Foot angle) & 0,02 & $0,01-0,08$ \\
\hline Inserção do úbere anterior (Fore udder attachment) & 0,10 & $0,09-0,14$ \\
\hline Altura do úbere posterior (Rear udder heigth) & 0,10 & $0,08-0,14$ \\
\hline Largura do úbere posterior (Rear udder width) & 0,14 & $0,08-0,18$ \\
\hline Ligamento central (Udder cleft) & 0,17 & $0,15-0,20$ \\
\hline Profundidade do úbere (Udder deep) & 0,17 & $0,15-0,20$ \\
\hline Colocação de tetas (Teat placement) & 0,29 & $0,23-0,32$ \\
\hline \multirow[t]{2}{*}{ Comprimento de tetas (Teat length) } & 0,16 & $0,15-0,18$ \\
\hline & \multicolumn{2}{|c|}{$\begin{array}{c}\text { Produção de leite } \\
\text { Milkyield } \\
\end{array}$} \\
\hline Produção de leite (Milk yield) & 0,22 & $0,18-0,25$ \\
\hline
\end{tabular}

${ }^{1}$ A partir das análises conjuntas de duas características.

${ }^{1}$ Starting from united analyses of two traits.

va de herdabilidade de 0,18 , demonstrando que esta característica poderia ser utilizada em programas de seleção, já que reflete o conjunto das características lineares e de classificação.

Quando são comparados os coeficientes de herdabilidade estimados, com os resultados obtidos por Gengler et al. (1999), também com animais da raça Pardo-Suíça, observa-se que as estimativas destes autores são levemente superiores às verificadas neste trabalho, principalmente para as características lineares profundidade corporal, abertura de garupa e tamanho de tetas. Estimativas de herdabilidade de outros estudos, como os de Short \& Lawlor (1992), Misztal et al. (1992) e Visscher \& Goddard (1995), na raça Holandesa, apresentaram resultados semelhantes.

No entanto, nos trabalhos de Weigel et al. (1998) e Esteves (1999), ambos com animais da raça Holandesa, as estimativas de herdabilidade foram superio- res as encontradas neste trabalho com animais da raça Pardo-Suíça, demonstrando que as estimativas de herdabilidade das características de conformação podem variar em função do sistema de classificação utilizado e da raça avaliada, como mostram os resultados obtidos por Gengler et al. (1999) avaliando cinco diferentes raças leiteiras (Pardo-Suíça; Jersey; Ayrshire; Guernsey; Milking Shorthorn).

As correlações genéticas e fenotípicas entre as características lineares de tipo, as características de classificação, a classificação final e a produção de leite podem ser verificadas na Tabela 6. De uma forma geral, as estimativas de correlações genéticas entre as características avaliadas foram superiores as estimativas de correlações fenotípicas correspondentes. Estes resultados estão de acordo com os resultados obtidos por outros autores (Klassen et al., 1992; Brotherstone, 1994).

As correlações fenotípicas entre as característi- 
cas lineares de capacidade corporal (estatura, força, profundidade e angulosidade) tenderam a apresentar correlações positivas moderadas. De forma semelhante, características lineares de sistema mamário (inserção anterior do úbere, altura e largura do úbere posterior, profundidade do úbere, ligamento central, colocação e comprimento de tetas) também tenderam a apresentar correlações positivas moderadas entre si. As características lineares de pernas e pés tenderam a apresentar correlações fenotípicas próximas de zero com as demais características, bem como entre si. As correlações fenotípicas verificadas entre as características de capacidade corporal e de sistema mamário também foram próximas de zero. Estes resultados estão de acordo com os obtidos por Misztal et al. (1992) e Esteves (1999).

As correlações genéticas entre as características lineares de tipo se apresentaram, em diversas ocasiões, altas. Foram verificadas altas correlações genéticas entre a estatura e as características lineares de garupa (ângulo e abertura de garupa), a angulosidade e as características lineares de sistema mamário (com exceção do comprimento de tetas), indicando que a seleção para animais altos indiretamente resulta em animais angulosos, com garupa ampla e ligeiramente baixa, e sistema mamário bem conformado. Gengler et al. (1999) verificaram resultados semelhantes com animais das raças Pardo-Suíça, Jersey e Guernsey.

Foram observadas correlações genéticas altas e positivas entre a angulosidade e as características de sistema mamário (com exceção da profundidade de úbere), indicando que vacas angulosas apresentaram sistema mamário bem inserido anteriormente, com úbere posterior alto e largo, ligamento central bem definido e tetas de comprimento desejável. De forma semelhante, também foram verificadas correlações genéticas de moderada a alta entre as características de sistema mamário (com exceção da largura do úbere posterior e do comprimento de tetas). Nos estudos de Short \& Lawlor (1992) e Gengler et al. (1999) foram observadas correlações genéticas semelhantes.

No entanto, foram observadas correlações genéticas negativas, de moderada a alta, entre a característica força e algumas características lineares de sistema mamário, como a altura e largura do úbere posterior, ligamento central e profundidade de úbere. No estudo de Esteves (1999) foram verificados resultados semelhantes, porém, no estudo de Misztal et al (1992) e Klassen et al. (1992), estes resultados não foram obtidos.

As características lineares corporais (estatura, força, ângulo e abertura de garupa) apresentaram correlações genéticas altas e negativas com a perna traseira (vista lateralmente), esta característica que, diferentemente, apresentou correlações genéticas altas e positivas com a maioria das características lineares de sistema mamário. Estes resultados mostram que vacas altas, fortes e com garupa alta e ligeiramente baixa apresentam pernas traseiras tendendo a retas, enquanto que vacas com sistema mamário bem conformado apresentam pernas traseiras, tendendo a curvas.

De forma semelhante, o ângulo do casco também apresentou correlações genéticas altas e negativas com as características corporais e de sistema mamário, indicando que a seleção para a melhoria destas características poderia resultar em vacas com ângulo de casco baixo. Os resultados já discutidos concordam com os obtidos por Esteves (1999) e Gengler et al. (1999).

As características de classificação usualmente não têm sido avaliadas em estudos de correlações genéticas e fenotípicas entre características de tipo e produção de leite. Neste estudo foram encontradas correlações genéticas e fenotípicas, de moderada à alta, entre as características de classificação e a maioria das características lineares, sendo estes resultados semelhantes aos obtidos por Visscher \& Goddard (1995).

Estas correlações genéticas e fenotípicas positivas corresponderam às características lineares que compõe a pontuação das características de classificação, segundo as regras oficiais de classificação da ABCGPS (ABCGPS, 2000). Em alguns casos, no entanto, também foram verificadas correlações genéticas e fenotípicas negativas entre estas características.

As correlações genéticas e fenotípicas entre as características de classificação foram altas, demonstrando que vacas que apresentaram altas pontuações para determinada característica de classificação, tendem a apresentar nas demais. De forma semelhante, a classificação final, resultado ponderado das características de classificação (ABCGPS, 2000), também apresentou elevadas correlações genéticas e fenotípicas com as características de classificação.

Em relação às correlações entre a classificação final e as características lineares de tipo, foram observadas correlações genéticas e fenotípicas de moderada a alta entre estas características, principalmente as características de sistema mamário e a estatura, demonstrando que vacas com pontuações 
RENNÓ et al.

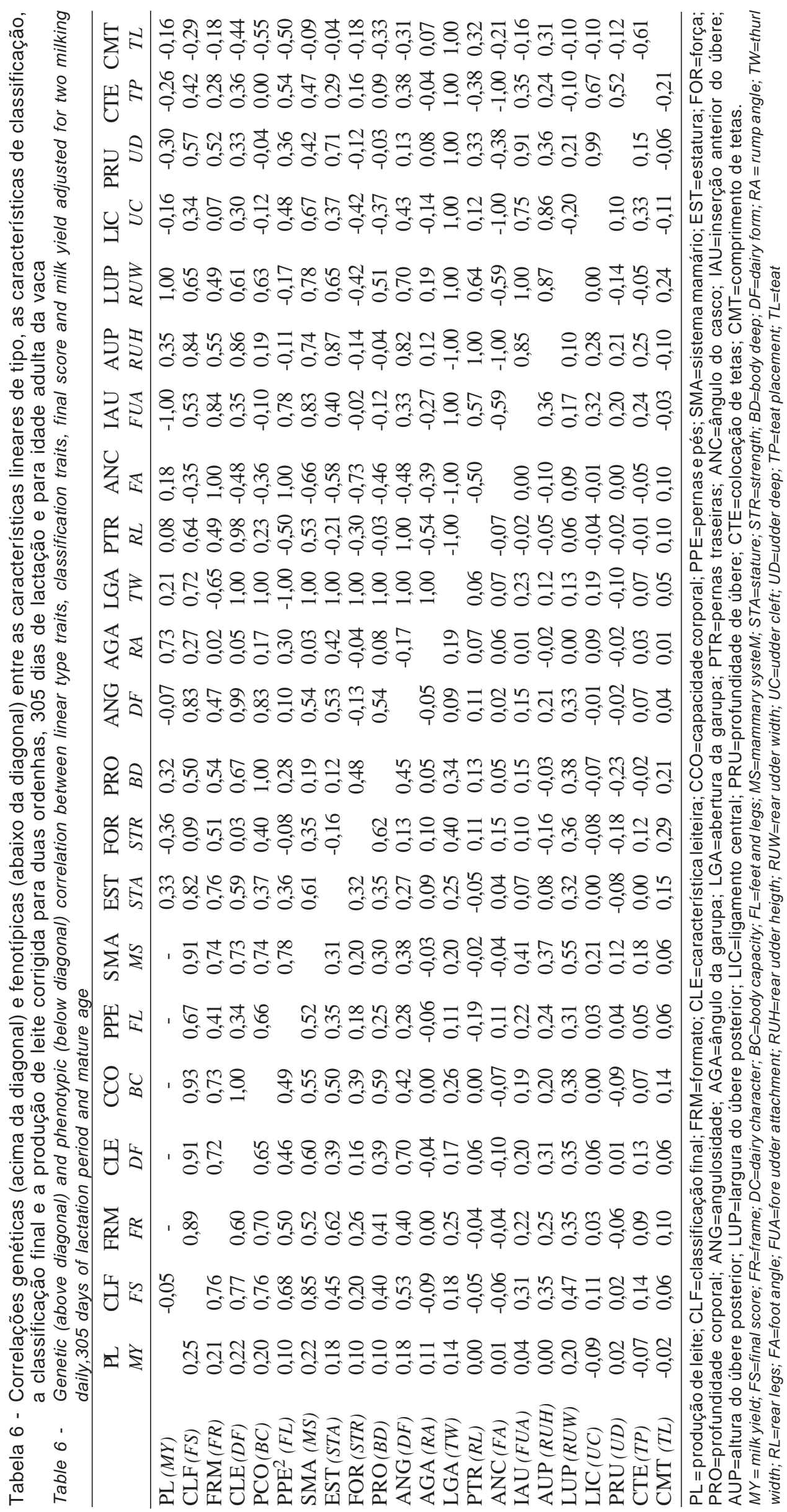


finais elevadas apresentam também, pontuações elevadas na maioria das características lineares. Estes resultados foram obtidos também nas avaliações realizadas por Short \& Lawlor (1992) e Esteves (1999).

As correlações fenotípicas entre as características lineares de tipo e a produção de leite geralmente apresentaram valores positivos próximos de zero, com as características largura de úbere posterior $(0,20)$, estatura $(0,18)$ e ângulo de garupa $(0,18)$ apresentando os maiores valores de correlação. No entanto, algumas características apresentaram correlações fenotípicas negativas, também com valores próximos a zero, com as características ligamento central $(-0,09)$ e colocação de tetos $(-0,07)$ apresentando os maiores valores.

As correlações fenotípicas entre as características de classificação e a classificação final com a produção de leite foram todas positivas, variando de 0,10 a 0,25 , com a classificação final apresentando o maior valor $(0,25)$, e a característica de classificação pernas e pés, o menor valor $(0,10)$.

Estes resultados são semelhantes aos obtidos por Norman et al. (1996) avaliando a raça Pardo-Suíça, obtendo as maiores correlações fenotípicas entre as características de tipo e a produção de leite com as características classificação final $(0,18)$, largura de úbere posterior $(0,11)$ e altura de úbere posterior $(0,09)$. Esteves (1999), avaliando a raça Holandesa, verificou que as características largura de úbere posterior $(0,16)$, classificação final $(0,15)$ e angulosidade $(0,14)$ apresentaram as maiores correlações fenotípicas com a produção de leite. Misztal et al. (1992) e Short \& Lawlor (1992) apresentaram os mesmos resultados.

As correlações genéticas entre as características lineares de tipo e a produção de leite foram usualmente maiores do que as correlações fenotípicas, estando de acordo com estudos descritos na literatura (Short \& Lawlor, 1992; Visscher \& Goddard, 1995). A maior correlação genética entre a produção de leite e as características lineares de tipo foram o úbere posterior (altura e largura), ângulo de garupa, estatura e profundidade corporal, demonstrando que vacas altas, com ampla abertura toráxica e abdominal, ângulo de garupa tendendo a baixo, e úberes posteriores largos e altos, tendem a apresentar maiores produções de leite. No entanto, as demais características de úbere, profundidade, inserção anterior, colocação e comprimento de tetas, e ligamento central, bem como a característica força, apresenta correlações genéticas negativas, de moderada a alta, com a produção de leite.

As correlações genéticas negativas obtidas entre as características de sistema mamário e a produção de leite revelam que vacas de maior produção de leite tendem a apresentar úberes mais profundos e fracamente inseridos, bem como com tetas de formato indesejado, demonstrando que quando a seleção para leite é aplicada sem restrições para tipo, efeitos indesejáveis sobre o sistema mamário poderão ocorrer. Desta forma, o comprimento da vida produtiva pode ser comprometido, com conseqüente redução da longevidade e aumento do descarte involuntário destes animais.

Misztal et al. (1992) concluíram que a seleção continuada para produção de leite causa deterioração em algumas características lineares de tipo, especialmente sobre as características do sistema mamário. Zwaag (1999), citado por Esteves (1999), salienta que a profundidade de úbere é a característica que leva a maior taxa de descarte em relação às demais, ou seja, úberes mais profundos são os maiores responsáveis pela exclusão de animais dos rebanhos.

Norman et at. (1988), Misztal et al (1992) e Esteves (1999) apresentaram resultados semelhantes, assim como Short \& Lawlor (1992) e Visscher \& Goddard (1995). Comparando os resultados obtidos neste estudo e os descritos anteriormente, não foi observada correlação genética expressiva entre a característica leiteira e a classificação final com a produção de leite, diferindo do comumente relatado.

\section{Conclusões}

Efeitos não-genéticos influenciam a avaliação da maioria das características de tipo, devendo ser considerados nas avaliações de conformação de bovinos desta raça.

As estimativas de herdabilidade obtidas sugerem a possibilidade de ganhos genéticos moderados advindos da seleção para características de tipo.

Devido às altas correlações genéticas entre as características lineares de tipo, programas de melhoramento para características de conformação podem ser implementados sem a necessidade de inclusão de todas as características.

As correlações genéticas observadas entre algumas características de tipo e a produção de leite sugerem a participação destas na formação de índices de seleção para animais desta raça. 


\section{Agradecimento}

À Associação Brasileira de Criadores de Gado Pardo-Suíço, pela cessão dos dados e pela oportunidade de desenvolver estudos com esta raça.

\section{Literatura Citada}

ALLAIRE, F.R.; GIBSON, J.P. Genetic value of herd life adjusted for milk production. Journal of Dairy Science, v.75, p.13491356, 1992.

ASSOCIAÇÃO BRASILEIRA DOS CRIADORES DE GADO PARDO-SUÍÇO. Regras Oficiais para Classificação Linear. ABCGPS, 2000. 11p.

BERTRAND, J.A.; BERGER, P.J.; FREEMAN, A.E. et al. Profitability in daughters of high versus average Holstein sires selected for milk yield of daughters. Journal of Dairy Science, v.68, p.2287-2294, 1985.

BOWDEN, V. Type classification in dairy cattle: A review. Animal Breeding. Abstract, v.50, n.3, p.147-161, 1982.

BOETTCHER, P.J.; DEKKERS, J.C.M.; KOLSTAD, B.W. Development of an udder health index for sire selection based on somatic cell score, udder conformation, and milking speed. Journal of Dairy Science, v.81, n.4, p.1157-1168, 1998.

BOLDMAN, K.G.; FREEMAN, A.E.; HARRIS, B.L. et al. Prediction of sire transmitting abilities for linear type traits. Journal of Dairy Science, v.75, n.2, p.552-563, 1992.

BOLDMAN, K.G.; KRIESE, L.A.; Van VLECK, L.D. et al. A manual for use of MTDFREML: a set of programs to obtein estimates of variances and covariances. Linconls: Department of Agriculture/Agricultural Research Service, 1995.120p.

BROTHERSTONE, S. Genetic and phenotypic correlation between linear type traits and production traits in Holstein-Friesian dairy cattle. Animal Production, v.59, n.2, p.183-187, 1994.

CAMPOS, M.S.; WILCOX, C.J.; BECERRIL, C.M. et al. Genetic parameters for yield and reproductive traits of Holstein and Jersey cattle in Florida. Journal of Dairy Science, v.77, n.3, p.867-873, 1994.

CUE, R.I.; HARRIS, B.L.; RENDEL, J.M. Genetic parameters for traits other than production in purebred and crossbred New Zealand dairy cattle. Livestock Production Science, v.45, n.2/3, p.123-135, 1996.

ESTEVES, A.M.C. Correlações genéticas e fenotípicas entre características lineares de tipo e produção de leite em rebanhos da raça Holandesa do estado de Minas Gerais. Belo Horizonte, MG: Universidade Federal de Minas Gerais, 1999.51p. Dissertação (Mestrado em Zootecnia) - Universidade Federal de Minas Gerais, 1999.

FREITAS, A. F.; DURÃES, M. C.; TEIXEIRA, N. M., et al. Estimativas de parâmetros genéticos para produção de leite e gordura de vacas Holandesas-PO no Estado de Minas Gerais. In: REUNIÃO ANUAL DA SOCIEDADE BRASILEIRA DE ZOOTECNIA, 36., 1999, Porto Alegre. Anais... Porto Alegre:SBZ, 1999. p.150.

GENGLER, N.; WIGGANS, G.R.; WRIGHT, J.R. Animal model genetic evaluation of type traits for five dairy cattle breeds. Journal of Dairy Science, v.82, n.6, p.1350-1368, 1999.

KLASSEN, D.J.; MONARDES, H.G.; JAIRATH, L. et al. Genetic correlations between lifetime production and linearized type in Canadian Holsteins. Journal of Dairy Science, v.75, n.8, p.2272-2282, 1992.

MATOS, R.S.; RORATO, P.R.N.; FERREIRA, G.B. et al. Estudo dos efeitos genéticos e de meio ambiente sobre a produção de leite e gordura da raça Holandês no Estado do Rio Grande do Sul. Ciência Rural, v.27, n.3, p.465-471, 1997.
McMANUS, C.; SAUERESSIG, M.G. Estudo de características lineares de tipo em gado Holandês em confinamento total no Distrito Federal. Revista Brasileira de Zootecnia, v.27, n.5, p.906-915, 1998.

MISZTAL, I.; LAWLOR, T.J.; SHORT, T.H. et al. Multiple-trait estimation of variance components of yield and type traits using an animal model. Journal of Dairy Science, v.75, n.2, p.544-551, 1992.

MRODE, R.A.; SWANSON, G.J.T. Genetic and phenotypic relationship between conformation and production traits in Ayrshire heifers. Animal Production, v.58, n.3, p.335-338, 1994.

NORMAN, H.D.; POWELL, R.L.; WRIGHT, J.R. et al. Phenotypic and genetic relationship between linear functional type traits and milk yield for five breeds. Journal of Dairy Science, v.71, p.1880-1896, 1988.

NORMAN, H.D.; POWELL, R.L.; WRIGHT, J.R. et al. Phenotypic relationship of yield and type scores from first lactation with herd life and profitability. Journal of Dairy Science, v.79, n.4, p.689-701, 1996.

RENNÓ, F.P. Aspectos produtivos da raça Pardo-Suíça no Brasil. Viçosa, MG: Universidade Federal de Viçosa, 2001. 100p. Dissertação (Mestrado em Zootecnia) - Universidade Federal de Viçosa, 2001.

RENNÓ, F.P.; PEREIRA, J.C.; ARAÚJO, C.V. et al. Estimativa de parâmetros genéticos para a produção de leite e gordura da raça Pardo-Suíça no Brasil. In: REUNIÃO ANUAL DA SOCIEDADE BRASILEIRA DE ZOOTECNIA, 39., 2001, Piracicaba. Anais... Piracicaba: Sociedade Brasileira de Zootecnia, 2001, p.535.

ROGERS, G.W.; McDANIEL, B.T. The usefulness of selection for yield and functional type traits. Journal of Dairy Science, v.72, p.187-193, 1989.

ROGERS, G.W.; McDANIEL, B.T.; DENTINE, M.R. et al. Genetic correlation between survival and linear type traits measured in first lactation. Journal of Dairy Science, v.72, p.523-527, 1989.

ROGERS, G.W.; VAN ARENDONK, J.A.M.; McDANIEL, B.T. Influence of involuntary culling on optimum culling rates and annualized net revenue. Journal of Dairy Science, v.71, p.3463-3469, 1988.

SAS INSTITUTE. SAS system for linear models. 3.ed., Cary: 1991.

SHORT, T.H.; LAWLOR, T.J. Genetic parameters of conformation traits, milk yield, and herd life in Holstein. Journal of Dairy Science, v.75, n.7, p.1987-1998, 1992.

THOMPSON, J.R.; LEE, K.L; FREEMAN, A.E. et al. Evaluation of a linearized type appraisal system for Holstein cattle. Journal of Dairy Science, v.66, n.2, p.325-331, 1983.

Van DORP, T.E.; DEKKERS, J.C.M.; MARTIN, S.W. Genetic parameters of health disorders, and relationship with 305-day milk yield and conformation traits of registered Holstein cows. Journal of Dairy Science, v.81, n.8, p.2264-2270, 1998.

Van VLECK, L.D.; NORMAN, H.D. Association of type traits with reasons for disposal. Journal of Dairy Science, v.55, n.12, p.1698-1705, 1972.

Van RADEN, P.M.; KLAASKATE, E.J.H. Genetic evaluation of length of productive life including predicted longevity of live cows. Journal of Dairy Science, v.76, p.2758-2764, 1993.

VISSCHER, P.M.; GODDARD, M.E. Genetic parameters for milk yield, survival workability, and type traits for Australian dairy cattle. Journal of Dairy Science, v.78, n.1, p.205-220, 1995.

WEIGEL, K.A.; LAWLOR Jr, T.J.; Van RADEN et al. Use of linear type traits and production data to supplement early predicted transmitting abilities for productive life. Journal of Dairy Science, v.81, n.7, p.2040-2044, 1998.

Recebido em: 31/05/02 Aceito em: 11/02/03 